\title{
Neuronal Glutamate Transporters Regulate Glial Excitatory Transmission
}

\author{
Ming-Chi Tsai, ${ }^{1}$ Kohichi Tanaka, ${ }^{2}$ Linda Overstreet-Wadiche, ${ }^{1}$ and Jacques I. Wadiche ${ }^{1}$ \\ ${ }^{1}$ Department of Neurobiology and Evelyn McKnight Brain Institute, University of Alabama at Birmingham, Birmingham, Alabama 35294 and ${ }^{2}$ School of \\ Biomedical Science and Medical Research Institute, Tokyo Medical and Dental University, Bunkyo-Ku, Tokyo 113-8510, Japan
}

In the CNS, excitatory amino acid transporters (EAATs) localized to neurons and glia terminate the actions of synaptically released glutamate. Whereas glial transporters are primarily responsible for maintaining low ambient levels of extracellular glutamate, neuronal transporters have additional roles in shaping excitatory synaptic transmission. Here we test the hypothesis that the expression level of the Purkinje cell (PC)-specific transporter, EAAT4, near parallel fiber (PF) release sites controls the extrasynaptic glutamate concentration transient following synaptic stimulation. Expression of EAAT4 follows a parasagittal banding pattern that allows us to compare regions of high and low EAAT4-expressing PCs. Using EAAT4 promoter-driven eGFP reporter mice together with pharmacology and genetic deletion, we show that the level of neuronal transporter expression influences extrasynaptic transmission from PFs to adjacent Bergmann glia (BG). Surprisingly, a twofold difference in functional EAAT4 levels is sufficient to alter signaling to BG, although EAAT4 may only be responsible for removing a fraction of released glutamate. These results demonstrate that physiological regulation of neuronal transporter expression can alter extrasynaptic neuroglial signaling.

\section{Introduction}

A family of five sodium-dependent glutamate transporter proteins [excitatory amino acid transporter 1 (EAAT1)-EAAT5] maintain the extracellular concentration of glutamate in the submicromolar range by taking up synaptically released glutamate into neurons and glia (for review, see Tzingounis and Wadiche, 2007). Knock-out (KO) studies in rodents show that glial transporters (EAAT1/GLAST and EAAT2/GLT-1) are responsible for recovering the majority of synaptically released glutamate and thus prevent glutamate excitoxicity (Rothstein et al., 1996; Tanaka et al., 1997; Huang et al., 2004b; Takayasu et al., 2005). Neuronal transporters appear to have additional functions. For example, the retinal transporter EAAT5 acts as a glutamate gated chloride channel to provide shunting inhibition (Veruki et al., 2006; Wersinger et al., 2006). Additionally, EAAT3, the transporter expressed by neurons throughout the brain, has been suggested to (1) transport cysteine for subsequent glutathione synthesis (Aoyama et al., 2006), (2) supply glutamate at inhibitory terminals for GABA synthesis (Sepkuty et al., 2002; Mathews and Diamond, 2003), and (3) limit glutamate spillover and favor NMDA receptor-dependent plasticity in the hippocampus (Diamond, 2001; Scimemi et al., 2009). These studies suggest that

Received Oct. 17, 2011; revised Dec. 5, 2011; accepted Dec. 18, 2011.

Author contributions: M.-C.T., L.O.-W., and J.I.W. designed research; M.-C.T. performed research; K.T. contributed unpublished reagents/analytic tools; M.-C.T., L.O.-W., and J.I.W. analyzed data; M.-C.T., L.O.-W., and J.I.W. wrote the paper.

This work was supported by National Institutes of Health Grant NS065920. We thank Anastassios V. Tzingounis and members of the Wadiche labs for discussions and critical reading of this manuscript.

Correspondence should be addressed to Linda Overstreet-Wadiche or Jacques I. Wadiche, Department of Neurobiology, SHEL 1005, University of Alabama at Birmingham, Birmingham, AL 35294. E-mail: Iwadiche@uab.edu or jwadiche@uab.edu.

DOI:10.1523/JNEUROSCI.5232-11.2012

Copyright $\odot 2012$ the authors $\quad 0270-6474 / 12 / 321528-08 \$ 15.00 / 0$ neuronal transporters contribute to multiple aspects of synaptic signaling as well as to the maintenance of low extracellular glutamate.

The differential functions of glial and neuronal transporters in the cerebellum have been studied extensively (for review, see Takayasu et al., 2009). Cerebellar glial transporters recover the majority of released glutamate (Brasnjo and Otis, 2004) and EAAT4, a high-affinity neuronal transporter expressed exclusively in Purkinje cells (PCs), limits activation of extrasynaptic glutamate receptors and/or silent synapses by recovering the residual glutamate (Brasnjo and Otis, 2004; Takayasu et al., 2004, 2005). These results are mostly derived from studies in $\mathrm{KO}$ animals that lack the EAAT4 protein or experiments using complete pharmacological blockade of transporters. The privileged perisynaptic localization of EAAT4, however, predicts that even subtle differences in EAAT4 expression levels could influence the activation of perisynaptic receptors by synaptically released glutamate (Dehnes et al., 1998; Wadiche and Jahr, 2005; Zheng et al., 2008).

In this study, we directly test the hypothesis that physiological differences of EAAT4 levels across parallel fiber (PF)-PC synapses controls the extrasynaptic glutamate concentration transient. We compare the extracellular glutamate transient produced by PF stimulation around high- and low-EAAT4expressing PCs using EAAT4 promoter-driven eGFP reporter mice. We find that the EAAT4 expression level alters the extracellular glutamate concentration profile to regulate the degree of Bergmann glia (BG) AMPA receptor (AMPAR) activation. We conclude that physiological differences of EAAT4 expression have functional consequences at PF-PC synapses, suggesting that endogenous regulation of transporter levels shape extrasynaptic glutamate signaling throughout the CNS. 


\section{Materials and Methods}

Animals. EAAT4-eGFP mice were acquired from the Mutant Mouse Regional Resource Center (MMRRC) at University of California, Davis (Davis, CA; MMRRC ID \#12845). The transgenic line was originally created by the National Institute of Neurological Disorders and Stroke GENSAT BAC Transgenics Project (Gong et al., 2003). EAAT4-KOeGFP mice were generated by crossing EAAT4-eGFP mice with EAAT4-KO mice (Huang et al., 2004a). To differentiate the BAC construct encoding the slc1a6 gene in EAAT4-eGFP mice from the endogenous slcla6 gene, EAAT4 genotypes were identified post hoc by reversetranscription PCR of total RNA extracted from cerebellar slices used for recording with specific primer sets (data not shown).

Slice preparation. Mice [postnatal day 13-20 (P13-P20)] of either sex were anesthetized by inhalation of isoflurane and killed by decapitation as approved by Institutional Animal Care and Use Committee of University of Alabama at Birmingham. Horizontal slices $(250-300 \mu \mathrm{m})$ from cerebellum were cut (Leica VT1200s; Leica Microsystems) in ice-cold cutting solution containing the following (in $\mathrm{mm}$ ): 110 choline $\mathrm{Cl}, 2.5$ $\mathrm{KCl}, 0.5 \mathrm{CaCl}_{2}, 7 \mathrm{MgCl}_{2}, 1.25 \mathrm{NaH}_{2} \mathrm{PO}_{4}, 25 \mathrm{NaHCO}_{3}, 25$ glucose, 11.5 $\mathrm{Na}$-ascorbate, and $3 \mathrm{Na}$-pyruvate (saturated with $95 \% \mathrm{O}_{2}$ and $5 \% \mathrm{CO}_{2}$, $\mathrm{pH}$ 7.4). Slices were incubated in artificial cerebrospinal fluid (ACSF) containing the following (in $\mathrm{mM}$ ): $119 \mathrm{NaCl}, 2.5 \mathrm{KCl}, 2.5 \mathrm{CaCl}_{2}, 1.3$ $\mathrm{MgCl}_{2}, 1 \mathrm{NaH}_{2} \mathrm{PO}_{4}, 26.2 \mathrm{NaHCO}_{3}$, and 11 glucose (saturated with $95 \%$ $\mathrm{O}_{2}$ and $5 \% \mathrm{CO}_{2}, \mathrm{pH} 7.4$ ) at $34^{\circ} \mathrm{C}$ for $30 \mathrm{~min}$ and then kept at room temperature (RT) for up to $5 \mathrm{~h}$.

Electrophysiology. Slices were visualized using a $60 \times$ water immersion objective on an Olympus BX51W upright microscope equipped with a contrast imaging gradient (Dodt et al., 2002), a mercury burner, and an eGFP filter set. Synaptic currents were recorded using a Multiclamp 700B amplifier and pClamp10 acquisition software (Molecular Devices), and then filtered at $2 \mathrm{kHz}$ and digitized at $10 \mathrm{kHz}$ using a Digidata 1440 analog-to-digital convertor (Molecular Devices). PCs and BG were identified by their morphological and electric properties and voltage clamped at -65 and $-75 \mathrm{mV}$, respectively. The intracellular solution for PC recordings contained the following (in $\mathrm{mm}$ ): $35 \mathrm{CsF}, 110 \mathrm{CsCl}, 20$ HEPES, and 10 CsEGTA, pH 7.3. For BG recordings, the intracellular solution contained the following (in mM): $82 \mathrm{CsCH}_{3} \mathrm{SO}_{3}, 40 \mathrm{CsCl}, 20$ HEPES, 10 HEDTA, and $3 \mathrm{BaCl}_{2}, \mathrm{pH}$ 7.3. Pipettes with an open tip resistance of 1.5-2 and 2-3 M $\Omega$ were used for PC and BG recordings, respectively. The recording chamber was perfused continuously with oxygenated ACSF at a rate of $\sim 3 \mathrm{ml} / \mathrm{min}$ at $30 \pm 2^{\circ} \mathrm{C}$ using an in-line heater (Warner Instrument). Picrotoxin (100 $\mu \mathrm{m}$; Sigma) was included in the ACSF to inhibit GABA $A_{A}$ receptors. We stimulated PFs with pairedpulse stimuli with 50-2000 ms interstimulus intervals (ISIs) to evoke EPSCs. Stimulating electrodes were made with borosilicate $\theta$ glass (BT150-10; Sutter Instrument) and placed 50-150 $\mu \mathrm{m}$ from target cells in the molecular layer. A constant voltage or current stimulus isolation unit $(20-150 \mu \mathrm{s})$ of $30-90 \mathrm{~V}$ or $(50 \mu \mathrm{s}) 50-300 \mu \mathrm{A}$, respectively, was used (Digitimer). Climbing fiber contamination was excluded by adjusting stimulation intensity and electrode location. To record synaptically evoked transporter currents (STCs) in PCs, the intracellular solution contained the following (in $\mathrm{mM}$ ): $130 \mathrm{CsNO}_{3}, 10$ tetraethylammoniumCl, 10 HEPES, 10 CsEGTA, 2 Mg-ATP, and 0.4 Na-GTP, pH 7.3. SR95531 ( $5 \mu \mathrm{M}$; Ascent), a competitive $\mathrm{GABA}_{\mathrm{A}}$ receptor antagonist, was included in the ACSF when $\mathrm{NO}_{3}{ }^{-}$-containing intracellular solution was used. PF-evoked STCs were isolated by applying NBQX (10 $\mu \mathrm{M})$ to block AMPARs without changing the stimulus intensity. The average PFevoked EPSC peak amplitudes in bright and dim PCs from Figure 2 were $488 \pm 58$ and $518 \pm 19 \mathrm{pA}(n=6$ each; $p>0.05)$, respectively. Bright and $\operatorname{dim}$ PCs did not differ in their input resistance $(76 \pm 13 \mathrm{M} \Omega$ and $92 \pm 19$ $\mathrm{M} \Omega$, respectively; $n=6$ each; $p>0.05$ ), and recordings had similar access resistances $(4.7 \pm 0.6 \mathrm{M} \Omega$ and $4.3 \pm 0.4 \mathrm{M} \Omega$, respectively; $n=6$ each; $p>0.05)$. PF-PC STCs were sensitive to DL-threo-betaBenzyloxyaspartate (DL-TBOA, $100 \mu \mathrm{M})$ inhibition $(82 \pm 6 \% ; n=4)$. In BG recordings, $100 \mu \mathrm{M} \mathrm{BaCl}$ and $100 \mu \mathrm{M}$ cyclothiazide (CTZ) were included in the ACSF to increase input resistance and enhance AMPA receptor responses, respectively. $\mathrm{BaCl}_{2}(100 \mu \mathrm{M})$ had no significant effect on three parameters of release: (1) the peak amplitude of climbing fiber- evoked PC STCs $(93.5 \pm 2.6 \%$ of control; $n=3 ; p>0.05)$, (2) the peak amplitude of PF-PC EPSCs (101.1 $\pm 3.6 \%$ of control; $n=6 ; p>0.05)$, and (3) the PF-PC EPSC paired-pulse ratio (PPR; $1.7 \pm 0.09$ and $1.7 \pm$ $0.08 ; 50 \mathrm{~ms}$ interstimulus interval; $n=6 ; p>0.05)$. To determine the contribution of the AMPAR $\left(I_{\mathrm{AMPA}}\right)$ component in the BG recordings, the BG-STC decay phase was subtracted from the decay of the PF-evoked responses [extrasynaptic currents (ESCs)] as in the paper by Matsui and Jahr (2003). The input-output curve (see Fig. 4A,C) was fitted with the equation $y(i)=\left(I_{\max }-I_{\min }\right) /\left(1+\exp ^{\left(i_{50}-i\right) / S}\right)$, where $y(i)$ is the current amplitude with intensity $i, i_{50}$ is the stimulus intensity yielding the half maximal response, and $S$ is the slope of the curve. We used the $20-80 \%$ of the EPSC rising phase for quantifying the rise time, except in Figure 4 we used the $50-90 \%$ of the EPSC rise to avoid contamination due to stimulation artifacts. Decay times were determined from a single exponential function.

All traces shown are the averages from 5 to 15 responses. Series resistance in PC recordings was compensated to $70-80 \%$. Recordings were excluded from analysis when the series resistance changed $>20 \%$ during the course of experiments. All chemicals were obtained from Sigma, Fisher Scientific, or Ascent Scientific unless indicated otherwise.

Immunohistochemistry and confocal imaging. EAAT4-eGFP mice (P17-P18) were perfusion fixed with $4 \%$ paraformaldehyde (Sigma), and $40 \mu \mathrm{m}$ horizontal cerebellar sections were sliced using a Vibratome S1000. Free-floating slices were treated with $3 \%$ hydrogen peroxide and $0.1 \%$ Triton X-100 in TBS ( $0.05 \mathrm{M}$ Tris, $0.15 \mathrm{M} \mathrm{NaCl}$, pH 7.4) for $20 \mathrm{~min}$ at RT and were then incubated with TBS containing 5\% normal goat serum (NGS) and $0.1 \%$ Triton X-100 for $1 \mathrm{~h}$ at RT. After blocking, slices were incubated to EAAT4 antiserum (1:50; a gift from Dr. J. Rothstein, Johns Hopkins Medical University, Baltimore, MD) in TBS with 2\% NGS and $0.1 \%$ Triton X-100 at $4^{\circ} \mathrm{C}$ for $48-60 \mathrm{~h}$. After three washes with TBS, slices were incubated with Alexa 568-conjugated secondary goat anti-rabbit antibody (1:500; Invitrogen) in TBS with $0.1 \%$ Triton X-100 for $1 \mathrm{~h}$ at RT. After wash of the second antibody, slices were mounted with anti-fade reagent (ProLong Gold; Invitrogen). Digital images were acquired using a $20 \times(\mathrm{NA}, 0.85)$ oil-immersion objective on an Olympus FluoView laser scanning confocal microscope using $488 \mathrm{~nm}$ and $568 \mathrm{~nm}$ excitation wavelengths for collecting eGFP and Alexa 568 fluorescence, respectively.

Image analysis. Confocal images were processed with ImageJ (NIH) and Photoshop software (Adobe). Fluorescent signals (eGFP and Alexa 568 ) from each slice were collected using a region of interest (ROI) from either a matched region (match) or from different regions for each signal (random). To calculate the fold difference between high- and lowEAAT4-expression regions, the average background intensity taken from the molecular layer of the slice was subtracted from the average fluorescence intensities of the ROI.

Data analysis and statistics. Recordings were analyzed off-line using Clampfit10 (Molecular Devices) and AxoGraph X (AxoGraph Scientific). Unless noted, two tailed Student's $t$-tests were performed using Excel (Microsoft) to determine statistical significance at the $p<0.05$ level. Curve fitting and ANOVA were performed using GraphPad Prism (GraphPad Software). Data are reported as the mean \pm SEM.

\section{Results}

\section{eGFP expression correlates with EAAT4}

We used a line of BAC transgenic mice with eGFP expression driven by the EAAT4 promoter (EAAT4-eGFP) to monitor EAAT4 expression in cerebellar slices. In these reporter mice, PCs showed a parasagittal banding pattern of eGFP expression in horizontal sections (Fig. 1A), consistent with the EAAT4 protein expression pattern (Nagao et al., 1997; Dehnes et al., 1998). To determine whether EAAT4-eGFP predicts endogenous protein expression levels, we compared eGFP expression with EAAT4 protein using EAAT4 antiserum (EAAT4Ab) (Fig. $1 B$ ). Superimposed images show parallel expression patterns (Fig. $1 C$ ). We measured the fluorescence intensities from eGFP and EAAT4 sections under an ROI (Fig. $1 C, D$ ) spanning the molecular layer that is rich with PC dendrites. The eGFP fluorescence (green) and 

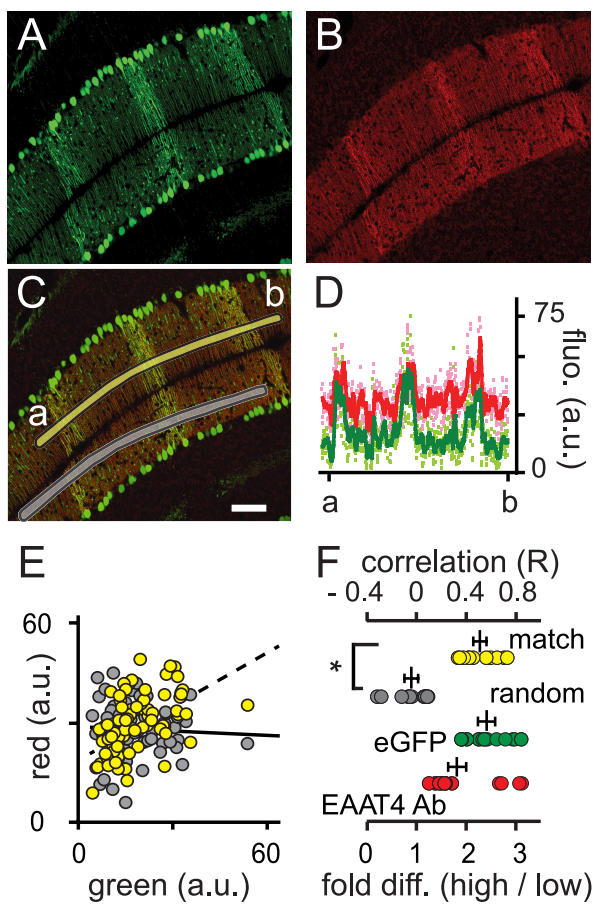

F correlation $(\mathrm{R})$
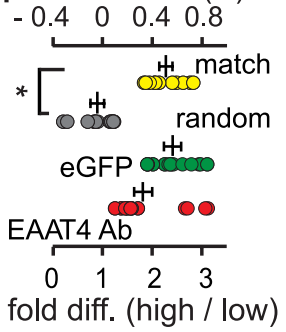

Figure 1. Expression of EAAT4-eGFP correlates with EAAT4. $\boldsymbol{A}$, GFP fluorescence confocal image from a transverse cerebellar slice of a P17 EAAT4-eGFP mouse. $\boldsymbol{B}$, Anti-EAAT4 staining from the same slice as in $\boldsymbol{A}$, revealed by Alexa 568 fluorescence. $\boldsymbol{C}$, Merged images from $\boldsymbol{A}$ and $\boldsymbol{B}$; yellow and gray regions are representative $\mathrm{RO}$ Is of the same shape and size used for subsequent analysis. Scale bar: $\mathbf{C}$ (for $\boldsymbol{A}-\boldsymbol{C}), 100 \mu \mathrm{m}$. D, Fluorescence measures in arbitrary units (a.u.) for eGFP (green) and EAAT4Ab (red) signals along the yellow ROl from point a to point $b$ in $C$; the dots are the actual values for each data point and the lines show a 10 point moving average. $\boldsymbol{E}$, Correlations of the EAAT4Ab versus the EGFP signals (yellow circles) both taken from the yellow ROI or the eGFP signal from the yellow ROI versus EAAT4Ab signal from the gray ROI (gray circles) from the image in $\boldsymbol{C}$. $\boldsymbol{F}$, Summary of correlation coefficients ( $R$; top graph) from match (yellow circles) and random groups (gray circles; $n=11$ each) as analyzed in $\boldsymbol{D}$. The bottom graph shows the fold difference between high and low expression regions of eGFP fluorescence (green) and EAAT4Ab signal (red), respectively ( $n=6$ slices), as analyzed in $\boldsymbol{D} .{ }^{*} p<0.05$.

the EAAT4Ab signal (red) were correlated within the same ROI (Fig. $1 C$, yellow ROI; $E$, yellow circles; $F$, match) $(r=0.49 \pm 0.04$; $n=11$ ). In contrast, the eGFP fluorescence (from the yellow ROI) was not correlated to the EAAT4Ab signal measured from a distinct area (Fig. $1 C$, gray ROI; $E$, gray circles; $F$, random) $(r=$ $-0.03 \pm 0.04 ; n=11 ; p<0.01)$. Additionally, there was a $2.4 \pm$ 0.2 -fold difference in eGFP fluorescence across bright and dim parasagittal bands (as measured from plots in Fig. $1 D$ ) similar to the $1.8 \pm 0.2$-fold difference measured with the EAAT4Ab signal (Fig. $1 D, F)(n=6$ slices). This difference in EAAT4 across parasagittal bands is consistent with previous reports using immunogold staining (Dehnes et al., 1998) and STCs from PCs in lobules representing high and low EAAT4 expression (Wadiche and Jahr, 2005).

\section{eGFP expression predicts functional EAAT4 activity}

We next determined the relationship between eGFP expression and functional EAAT4 activity by measuring synaptic transporter currents (STCs) from PCs with high and low eGFP expression, hereafter referred to as bright and dim PCs, respectively. Guided by visual eGFP expression (Fig. 2A1,A2), we recorded PF AMPAR-mediated (PF-PC) EPSCs with stimulus intensities adjusted to keep the EPSCs below $500 \mathrm{pA}$. Without changing the stimulus intensity, we then added NBQX $(10 \mu \mathrm{M})$ to block AMPARs (Fig. 2 B1,B2). Consistent with the differential expression of
EAAT4, the average peak amplitude of STCs was $\sim 50 \%$ greater in bright PCs compared to dim PCs (STC, $14.2 \pm 1.6 \mathrm{pA}$ vs $9.5 \pm 1.7$ $\mathrm{pA} ; n=6 ; p<0.05$ ) (Fig. $2 B 1-D$ ). Even when we normalized for the stimulus strength across recordings, we found that the ratio of the STC amplitude to the EPSC amplitude was larger in bright PCs compared to dim PCs $\left(\mathrm{STC}_{1} / \mathrm{EPSC}_{1}=0.014 \pm 0.003\right.$ vs $0.008 \pm 0.001 ; n=6 ; p<0.05$ ) (Fig. $2 E$ ). As expected, the STC decay time constant (evoked with paired-pulse stimulation) was similar in bright and dim PCs (Fig. $2 F, G$ ) (bright vs dim, $26.5 \pm$ 2.3 vs $26.0 \pm 2.8 \mathrm{~ms}$; one exponential; $p>0.05 ; n=6$ each), since the STC response reflects intrinsic transporter kinetics independent from the rate of glutamate clearance (Wadiche et al., 2006). Together, these results show that expression of eGFP correlates with EAAT4 expression and function, thereby providing a model to test physiological consequences of endogenous variability in EAAT4 levels.

\section{Presynaptic properties at PF synapses of high- and low-EAAT4-expressing PCs}

Electrophysiological and anatomical studies show that mossy fibers originating from different nuclei are spatially separated and innervate granule cells in different Zebrin II-labeled zones (Voogd et al., 2003; Apps and Hawkes, 2009). This raises the possibility that PF release sites may also differ between high- and low-EAAT4-expressing zones, as recently suggested for climbing fibers (Paukert et al., 2010; but see Wadiche and Jahr, 2005). To assess PF presynaptic properties at bright and dim PCs, we compared the PF-evoked peak synaptic glutamate concentration using the low affinity, competitive glutamate receptor antagonist kynurenate (KYN) (Clements, 1996). We found that $1 \mathrm{mM} \mathrm{KYN}$ inhibited the EPSC measured from bright and dim PCs to the same extent $\left(\mathrm{EPSC}_{1}, 70 \pm 4 \%\right.$ vs $70 \pm 6 \%$ EPSC $_{2}, 60 \pm 4 \%$ vs $61 \pm 7 \% ; p>0.05 ; n=6$ and 5 , respectively) (Fig. $3 A, B, D$ ), but affected the PPR (Fig. 3C) as expected (Foster et al., 2005). After washout of KYN, the high-affinity glutamate receptor antagonist NBQX (200 nM) blocked PF EPSCs to the same degree in bright and $\operatorname{dim}$ PCs $\left(\right.$ EPSC $_{1}, 72 \pm 2 \%$ vs $70 \pm 3 \%$ EPSC $_{2}, 70 \pm 2 \%$ vs $68 \pm 3 \% ; p>0.05 ; n=6$ and 5 , respectively) (Fig. $3 A, B, D$ ) without changing the PPR (Fig. $3 C$ ). The PPR was the same between bright and $\operatorname{dim}$ PCs $(1.8 \pm 0.1$ vs $1.9 \pm 0.1$ at $50 \mathrm{~ms}$ interstimulus interval; $p>0.05 ; n=6$ and 5 , respectively) (Fig. $3 C)$ at all ISIs tested between 10 and $2000 \mathrm{~ms}(n=8$ bright and $n=7 \mathrm{dim}$; ANOVA; $p>0.05$ ) (Fig. $3 E, F$ ). Similarly, the decay time constants of PF EPSCs from bright and dim PCs were not different (bright vs dim, single exponential time constant, $4.7 \pm$ 0.6 vs $4.3 \pm 0.4 \mathrm{~ms} ; p>0.05 ; n=6$ and 6 , respectively). Together, these results suggest that PF terminals across regions with different EAAT4 expression levels have similar release properties and that the variation of endogenous EAAT4 expression is not sufficient to alter the time course of EPSCs.

\section{Differences in glutamate spillover between regions of high and low EAAT4}

Endogenous variation in EAAT4 was not sufficient to alter PC EPSCs (Fig. 3) nor the recruitment of additional parallel fibers with increased stimulation intensity (Fig. $4 A$ ), suggesting that synaptic receptors were unaffected. However, the perisynaptic localization of EAAT4 is thought to restrict activation of extrasynaptic receptors by glutamate that escapes uptake by glial transporters (Dehnes et al., 1998; Takayasu et al., 2005; Wadiche and Jahr, 2005). Therefore, we wondered whether EAAT4 localization and density is sufficient to differentiate extrasynaptic receptor activation between synapses at dim and bright PCs. We used 

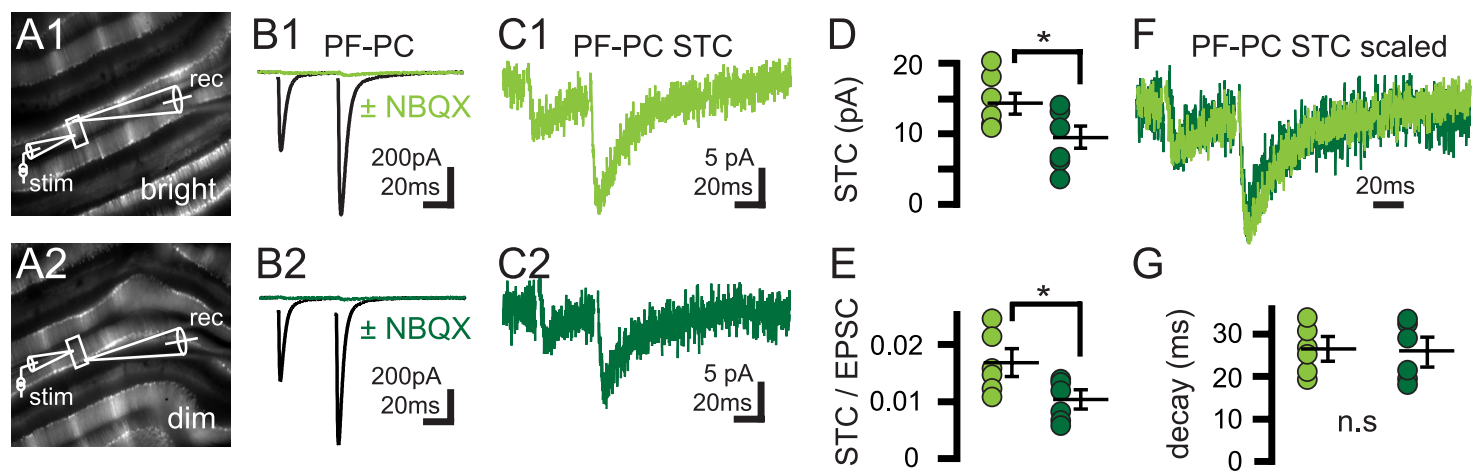

Figure 2. Functional EAAT4 level parallels with GFP level. $\boldsymbol{A}$ 1, $\boldsymbol{A 2}$, Fluorescence images taken during recordings made from bright (A1) and dim (A2) PCS. B1, B2, Corresponding PF evoked EPSC (black, before NBQX application) and STC (green, after NBQX application) traces recorded from bright (B1) and dim (B2) PCS. C1, C2, Enlarged STC traces from B1 and B2, respectively. D, Summary of the absolute value of the STC amplitudes. E, Summary of the ratio of the STC amplitude to the EPSC amplitude, used to account for stimulus intensities. F, Scaled traces from C1 and $\boldsymbol{C 2}$. G, Summary of the STC decay time constant. ${ }^{*} p<0.05$.

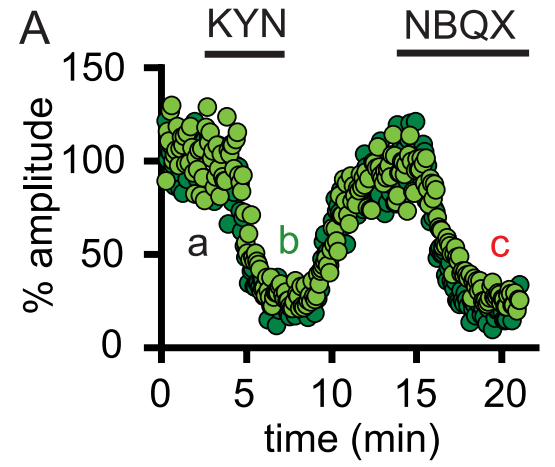

C

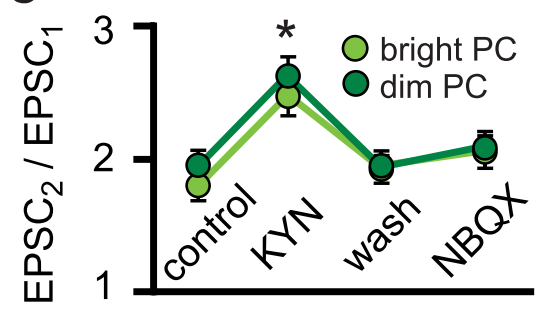

$\mathrm{E}$ interstimulus interval (ms)

$10,30,50,100,300,1000,3000$

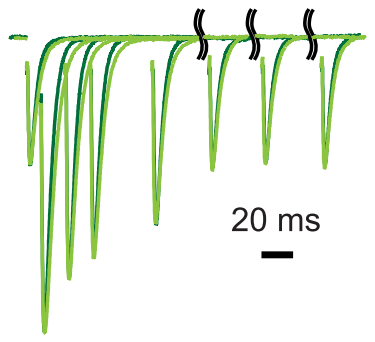

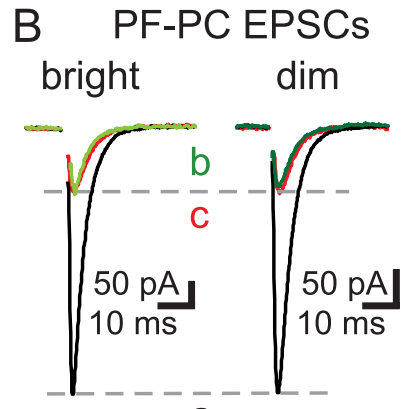

a
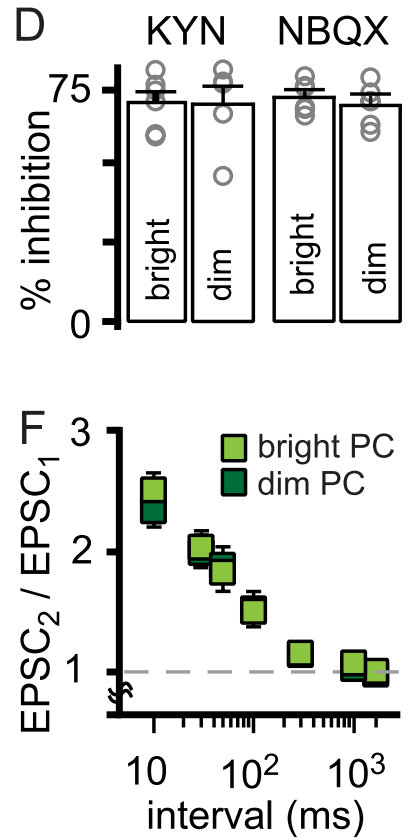

Figure 3. Parallel fiber release probability is the same between bright and $\operatorname{dim} P(S . A$, EPSC amplitudes during the application of KYN and NBQX for representative bright and dim PC recordings. $B$, The corresponding EPSC traces in control (a, black), 1 mm KYN (b, green), and $200 \mathrm{~nm} \mathrm{NBQX} \mathrm{(c,} \mathrm{red)} \mathrm{from} \boldsymbol{A}$ of bright (light green) and dim (dark green) PCs. C, Summary of PPR in different conditions for bright $(n=6)$ and $\operatorname{dim}(n=5)$ PCS. D, Summary of the percentage of inhibition by KYN and NBQX.E, Representative PF-PC EPSC traces evoked with different ISIs from bright and dim PCS. Traces are scaled to their first response. F, Summary of PPR at different ISIs from bright and dim PCs. ${ }^{*} p<0.05$.

CTZ $(100 \mu \mathrm{M})$ to promote the activation of extrasynaptic PC AMPARs via prevention of AMPAR desensitization and enhanced affinity (Matsui and Jahr, 2003). On average, CTZ increased the EPSC peak amplitude by $\sim 30 \%$ and slowed the EPSC kinetics to the same extent between bright and $\operatorname{dim}$ PCs (Fig. $4 B$; Table 1$)(p<0.05$; $n=10)$. Activation of extrasynaptic receptors contributes to enhanced EPSCs in CTZ. In addition, CTZ did not alter the PPR (Table 1$)(p>0.05 ; n=10)$. However in CTZ, comparison of EPSC peak amplitudes in bright and dim PCs as a function of stimulus intensities revealed that the current required to evoke a halfmaximum response $\left(i_{50}\right)$ was significantly greater in bright PCs $\left(i_{50}=178 \pm 6.7 \mu \mathrm{A}\right.$ vs $155.8 \pm 5.7 \mu \mathrm{A} ; p<0.05 ; n=6$ and 8 , respectively) (Fig. 4C). This difference may reflect easier activation of extrasynaptic AMPARs in PCs with lower EAAT4 expression and is consistent with the role of EAAT4 in limiting extrasynaptic receptor activation (Wadiche and Jahr, 2005; Nikkuni et al., 2007).

\section{EAAT4 regulates extrasynaptic}

glutamate concentration

To directly test the idea that EAAT4 expression levels regulate extracellular glutamate dynamics, we measured synaptic responses in BG that ensheath PC synapses. We recorded responses from BG surrounding bright and dim PCs (hereafter referred to as bright and dim regions). The PF-evoked extrasynaptic current (ESC) in BG is composed of an AMPA receptormediated current $\left(I_{\mathrm{AMPA}}\right)$ and a glutamate transporter-mediated current (BG STC) (Bergles et al., 1997; Clark and Barbour, 1997). The BG AMPAR response is predominantly due to ectopic release, but CTZ enables BG AMPARs to respond to lower and prolonged glutamate transients provided by spillover (Matsui and Jahr, 2003). We found that the low-affinity antagonist KYN (1 mM) (Fig. 3) blocked the BG ESC in bright regions to a greater degree compared to dim regions (block in bright vs dim, $\mathrm{ESC}_{1}, 62 \pm 2 \%$ vs $53 \pm 1 \%$; $\mathrm{ESC}_{2}$, $60 \pm 1 \%$ vs $52 \pm 2 \% ; p<0.05 ; n=6$ and 4 , respectively) (Fig. 


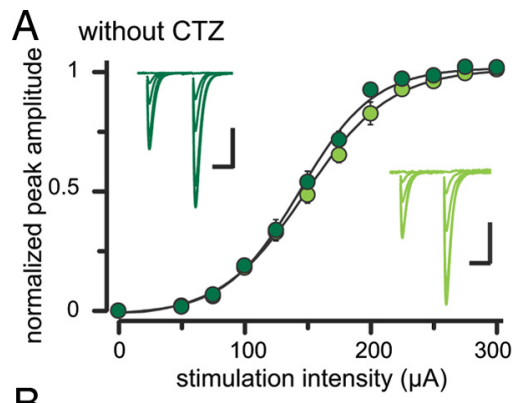

B
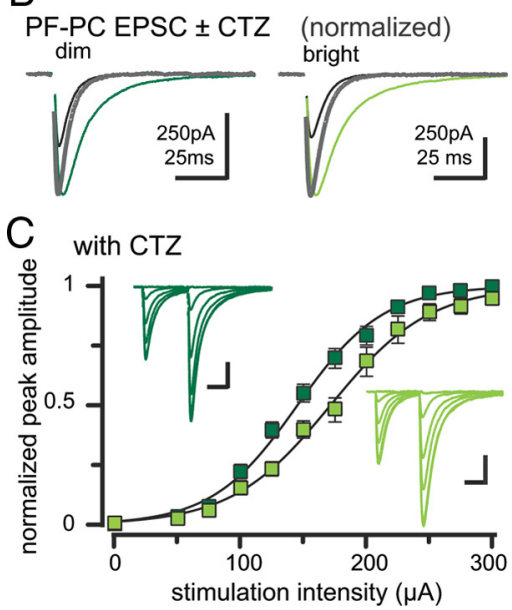

Figure 4. Altered extrasynaptic AMPA receptor activation in dim and bright PCS. A, Normalized peak amplitudes of the first EPSC from dim (dark green) and bright (light green) PCs. Responses are normalized to those evoked with maximal stimuli. The solid line is the best fit to the equation $y(i)=\left(I_{\max }-I_{\min }\right) /\left(1+\exp \wedge\left[\left(i_{50}-i\right) / S\right]\right)$, with $i_{50}=150.6 \pm 3.3 \mu \mathrm{A}$ vs $143.8 \pm 4.4 \mu \mathrm{A}$ and $S=30.5 \pm 2.8$ and $26.3 \pm 1.2$ for a group of dim and bright cells, respectively ( $n=5$ each; $p>0.05$ for both $i_{50}$ and $S$ ). Similar results were obtained with the second EPSC of a pair (data not shown). Inset, Representative PF-PC EPSCs evoked with two pulses. Overlaid traces are the EPSCs with increasing stimulus strengths in dim and bright PCs. $\boldsymbol{B}$, PF-PC EPSCs from dim and bright PCs before (black) and after the application of CTZ (100 $\mu \mathrm{M}$; dark or bright green) and the peak amplitude scaled (gray). C, Normalized EPSC peak amplitudes of the first EPSCs from bright and dim PCs in the presence of CTZ $(100 \mu \mathrm{m})$. The solid line is the best fit to the equation $y(i)=\left(I_{\max }-I_{\text {min }}\right) /\left(1+\exp \wedge\left[\left(i_{50}-i\right) / S\right]\right)$, with $i_{50}=178 \pm$ $6.7 \mu$ Avs $155.8 \pm 5.7 \mu$ A and $S=36.7 \pm 4$ and $33.5 \pm 2.5$ for a group of bright and dim cells, respectively $\left(p<0.05\right.$ for $i_{50} ; p>0.05$ for $\left.S\right)$. Similar results were obtained with the second EPSC of a pair (data not shown). Inset, Representative PF-PC EPSCs evoked with two pulses. Overlaid traces are the EPSCs in the presence of CTZ evoked with increasing stimulus strengths. Calibration: A, C, 500 pA, 25 ms.

Table 1. Summary of CTZ effects on PF-PC EPSCs

\begin{tabular}{|c|c|c|c|}
\hline & Control & CTZ & $\%$ change \\
\hline \multicolumn{4}{|l|}{ Amplitude (pA) } \\
\hline Bright $(n=6)$ & $-375.4 \pm 44.8$ & $-494.3 \pm 73.6$ & $31.0 \pm 13.5$ \\
\hline $\operatorname{Dim}(n=6)$ & $-417.9 \pm 73.5$ & $-523.3 \pm 57.2$ & $32.1 \pm 17.1$ \\
\hline \multicolumn{4}{|l|}{ Rise time (ms) } \\
\hline Bright $(n=6)$ & $0.91 \pm 0.05$ & $1.52 \pm 0.11$ & $68.7 \pm 11.6$ \\
\hline $\operatorname{Dim}(n=4)$ & $0.76 \pm 0.06$ & $1.27 \pm 0.08$ & $70.1 \pm 9.2$ \\
\hline \multicolumn{4}{|l|}{ Decay time (ms) } \\
\hline Bright $(n=6)$ & $5.75 \pm 0.3$ & $14.2 \pm 1.2$ & $146.7 \pm 19.4$ \\
\hline $\operatorname{Dim}(n=4)$ & $5.41 \pm 0.4$ & $14.2 \pm 0.7$ & $163.9 \pm 14.0$ \\
\hline \multicolumn{4}{|l|}{ PPR (ISI, 150 ms) } \\
\hline Bright $(n=6)$ & $1.4 \pm 0.04$ & $1.4 \pm 0.06$ & \\
\hline $\operatorname{Dim}(n=6)$ & $1.4 \pm 0.08$ & $1.4 \pm 0.05$ & \\
\hline
\end{tabular}

$5 A, C)$. In contrast, the high-affinity antagonist NBQX $(200 \mathrm{nM})$ blocked BG ESCs similarly in both regions (bright vs dim; $\mathrm{ESC}_{1}$, $48 \pm 2 \%$ vs $48 \pm 4 \%$; ESC $_{2}, 48 \pm 2 \%$ vs $50 \pm 3 \% ; p>0.05 ; n=$ 6 and 4 , respectively) (Fig. $5 A, C$ ). Differential block by KYN but
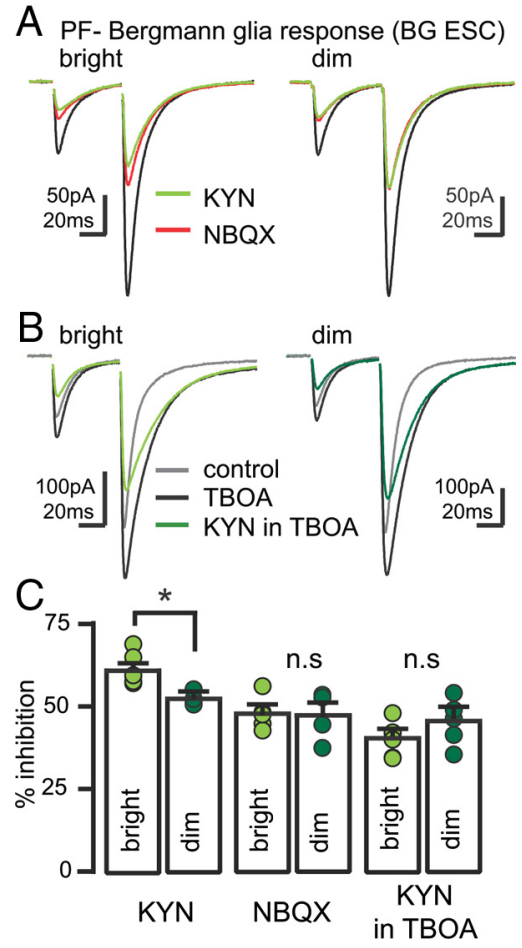

Figure 5. EAAT4 alters the glutamate concentration transient on Bergmann glia. $A, P F-B G$ responses (BG ESC) before (black) and after (green) application of KYN (1 mM) or NBQX (200 nм; red traces). B, PF-BG responses before (gray) and after (black) application of DL-TBOA $(50 \mu \mathrm{M})$. Inhibition by KYN (in TBOA) is shown in light and dark green for recordings from bright and dark regions, respectively. C, Summary of the percentage of inhibition by KYN and NBQX and KYN with TBOA. n.s., Not statistically significant. ${ }^{*} p<0.05$.

not NBQX indicates that the extrasynaptic glutamate transient is lower in regions of high EAAT4 expression.

In the presence of DL-TBOA $(50 \mu \mathrm{M})$, a nonselective glutamate transporter antagonist, the differential sensitivity to KYN was abolished. In TBOA, KYN (1 mM) blocked the BG ESC 1 in bright regions by $40 \pm 2 \%$ compared to $45 \pm 3 \%$ in dim regions $(p>$ $0.05 ; n=6$ and 5 , respectively; $28 \pm 1 \%$ compared to $31 \pm 5 \%$ at $\mathrm{ESC}_{2} ; p>0.05$ ) (Fig. $5 \mathrm{~B}, \mathrm{C}$ ). The reduced inhibition of ESCs in the presence of DL-TBOA is consistent with a higher extrasynaptic glutamate concentration when transporters are blocked. Since other glutamate transporter subtypes are homogeneously expressed (Dehnes et al., 1998), these data suggest that the regional difference in the extrasynaptic glutamate concentration is a result of EAAT4 expression and that a higher glutamate concentration transient is present in regions with low EAAT4 levels.

\section{EAAT4 controls the activation of Bergmann glia AMPA receptors}

We next asked whether the EAAT4-dependent extrasynaptic glutamate concentration differences are sufficient to affect AMPAR activation on BG. First we determined the contribution of AMPARs $\left(I_{\mathrm{AMPA}}\right)$ (Fig. $6 A, B$, green traces) and glutamate transporters (red traces) to the ESC (black traces) (see Material and Methods). To compare results across cells, we used the charge integral of the BG STCs $\left(Q_{\mathrm{STC}}\right)$ as an estimate of the number of glutamate molecules sensed by each BG. We found that the ratio of $I_{\mathrm{AMPA}}$ amplitude to $Q_{\mathrm{STC}}$ in bright regions was significantly smaller than in dim regions (bright vs dim, ratio, $0.10 \pm 0.01$ vs $0.21 \pm 0.03 ; p<0.05 ; n=10$ and 11 , respectively) (Fig. 6A,C). This suggests less activation of BG AMPA receptors in regions 
$B G E S C=B G S T C+B G \mid A M P A$
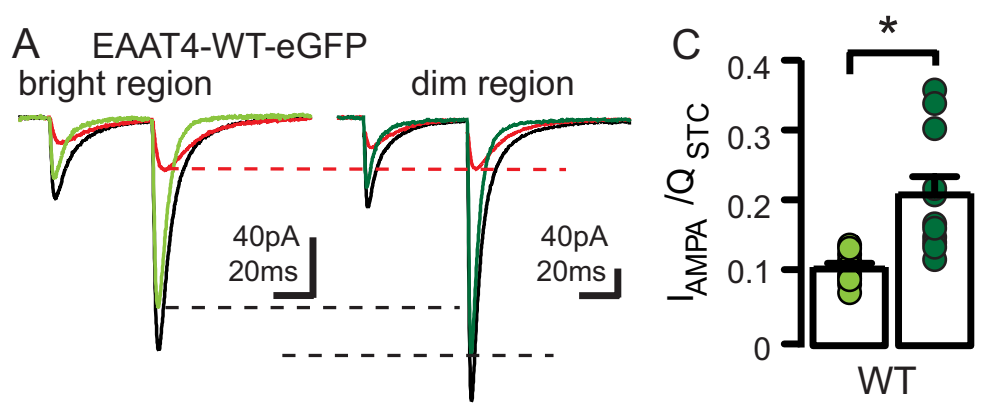

B EAAT4-KO-eGFP
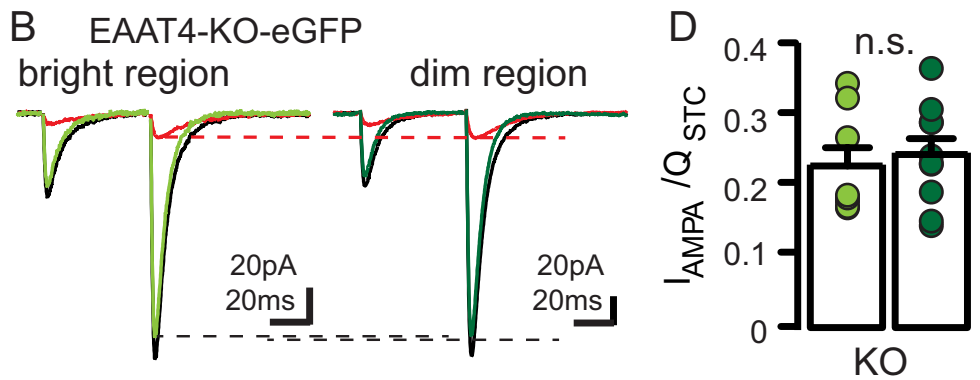

Figure 6. EAAT4 regulates Bergmann glial AMPAR activation. $\boldsymbol{A}, \boldsymbol{B}$, Representative BG ESC from EAAT4-WT-eGFP $(\boldsymbol{A})$ and EAAT4-K0-eGFP mice $(\boldsymbol{B})$ are the sum of BG $I_{\text {AMPA }}$ (green) plus BG STC (red). Recordings with light and dark green traces designate BGs located in bright and dim regions, respectively. BG responses in bright regions showed a reduced $I_{\text {AMPA }}$ component $(\boldsymbol{A}$, difference in black dashed lines) in WT mice but not in EAAT4 KO mice (B, dashed black lines). $\boldsymbol{C}, \boldsymbol{D}$, Summary results from EAAT4-WT-eGFP and EAAT4-KO-eGFP lines. n.s., Not statistically significant. ${ }^{*} p<0.05$.

with higher EAAT4 expression presumably due to the concentration differences (Fig. 5). To confirm the causal role of EAAT4 and control for potential differences in recording locations that could each regulate BG AMPARs, we crossed a mouse line with EAAT4 genetically deleted (EAAT4-KO) (Huang et al., 2004a) with the EAAT4-eGFP mice. The offspring progeny (EAAT4-KO-eGFP) allowed us to use eGFP to unambiguously identify regions of high and low EAAT4 expression in the absence of EAAT4 protein. In contrast to the results with EAAT4-WT-eGFP mice, there was no difference in BG AMPAR activation between bright and dim regions in EAAT4-KO-eGFP mice (bright vs dim, ratio, $0.22 \pm 0.03$ vs $0.24 \pm 0.02 ; p>0.05$ for both; $n=8$ and 10, respectively) (Fig. $6 B, D)$. These results strongly support the conclusion that EAAT4 regulates $B G$ AMPAR activation.

\section{Discussion}

Here we show that EAAT4 expression in PCs is inversely correlated with the degree of extrasynaptic glutamate receptor activation, the concentration of extracellular glutamate, and the degree of BG AMPA receptor activation following PF stimulation. We further show that blocking glutamate transporters or genetically removing EAAT4 from PCs abolished the regional difference in extracellular glutamate concentration, indicating that the regional differences in EAAT4 played a causal role. The observation that EAAT4 influences BG AMPA receptor activation despite the presence of abundant glial transporters (Lehre and Danbolt, 1998) suggests that the perisynaptic localization of neuronal transporters (Dehnes et al., 1998) endows EAAT4 with a privileged role in modulating neuroglia glutamate signaling. In sum, we demonstrate that physiologically relevant variation in neuronal glutamate transporter expression levels is sufficient to alter extrasynaptic glutamate signaling.
A system to study EAAT4 and cerebellar cortical compartmentalization

We established a system to study neuronal transporter function using EAAT4-eGFP mice. The differential expression of EAAT4 in PCs is faithfully represented by the eGFP signal. High and low eGFP expression also predict the level of endogenous EAAT4 functional activity. This system provides an assay to study physiological variations in neuronal transporter expression more subtle than complete loss of transporters using EAAT4-KO mice or pharmacological blockade. We also overcome the lack of a specific EAAT4 antagonist by generating EAAT4-KO-eGFP mice to validate the causal role of EAAT4 in our results. The patterned expression of EAAT4 provides the opportunity to understand the consequences of altered transporter expression levels in the absence of other pathological or compensatory changes that likely accompany transporter dysregulation in disease states (Serra et al., 2004, 2006; Ikeda et al., 2006). Furthermore, EAAT4-eGFP mice may be a useful tool in studying the functional significance of cerebellar compartmentalization, given that other proteins, such as protein kinase $\mathrm{C} \delta$ and $\mathrm{GABA}_{\mathrm{B} 2}$ receptor, are also positively or negatively correlated with EAAT4 expression (Apps and Hawkes, 2009).

\section{Parallel fiber release properties in high- and low-EAAT4-expressing zones}

Mossy fiber afferents terminate in longitudinal stripes that overlap with Zebrin II strips. Although granule cells relay information from mossy fibers, their parallel fiber axons often extend over the stripe boundaries (Apps and Hawkes, 2009). Our results indicate that PFs innervating high- and low-EAAT4-expressing PCs show similar release properties, unlike a recent report showing that climbing fibers have different release properties across Zebrin II and EAAT4 stripes (Paukert et al., 2010; but see Wadiche and Jahr, 2005). This result supports our conclusion that EAAT4 mediates the region-specific regulation of extrasynaptic glutamate concentration, ruling out the possible confounds in presynaptic release differences.

\section{EAAT4 modulates the extrasynaptic glutamate profile and receptor activation}

We found that in the presence of CTZ, EAAT4 levels influenced the recruitment of extrasynaptic AMPA receptors although they did not influence the PF-PC EPSC time course. We speculate that EAAT4 may control the degree of glutamate "spilling out" of synapses, resulting in the differential recruitment of extrasynaptic AMPA receptors. However, our data also suggest that even low levels of EAAT4 are sufficient to prevent "spillover" between synapses that contributes to the late decay phase of PF EPSCs seen in recordings from EAAT4 KO slices (Tsukada et al., 2005).

Together, these results show that EAAT4 plays an important role in restricting the activation of extrasynaptic glutamate receptors on PCs and BG, in addition to metabotropic glutamate receptors (mGluRs) (Brasnjo and Otis, 2001; Wadiche and Jahr, 
2005; Nikkuni et al., 2007). In this regard, neuronal transporters in the cerebellum are similar to EAAT3 in the hippocampus that limit extrasynaptic NMDA receptor activation (Scimemi et al., 2009).

Our results indicate that upon PF activation, physiological differences in EAAT4 expression can affect the extrasynaptic but not the synaptic glutamate concentration. The synaptic glutamate transient at cerebellar synapses depends primarily on the number and synchrony of released vesicles (Foster et al., 2005; Rudolph et al., 2011) that did not differ between regions. Neuronal EAATs could prolong the extrasynaptic glutamate transient by temporarily trapping glutamate without transport as well as reducing released glutamate by uptake (Diamond and Jahr, 1997; Tzingounis and Wadiche, 2007). EAAT4 is responsible for $\sim 20 \%$ of glutamate uptake following climbing fiber activation (Brasnjo and Otis, 2004), a value that could be higher in high-EAAT4expression PCs. We found that the BG STC rise time in highEAAT4 regions was significantly slower than dim regions in EAAT4-wild-type (WT) mice and both regions in EAAT4-KO mice (data not shown), potentially revealing enhanced buffering in high-EAAT4 regions. However, we cannot rule out the possibility that enhanced uptake capacity in bright regions contributes to the time course as well as the peak concentration of glutamate that reaches BG AMPA receptors.

\section{Functional implication of differential activation of BG AMPAR}

Imaging in vivo from awake animals suggests that suppressing glutamatergic signaling inhibits calcium transients in BG that are activated by motor behavior (Nimmerjahn et al., 2009). AMPARs on BG play a minor role in inducing the calcium signals (Piet and Jahr, 2007), but calcium signals mediated by BG mGluRs may also be influenced by EAAT4 levels. Furthermore, BG AMPA receptor activation is needed to maintain $\mathrm{BG}$ ensheathment of $\mathrm{PC}$ synapses (Iino et al., 2001; Ishiuchi et al., 2001). Therefore, differences in the synaptic activation of BG AMPA receptor between high- and low-EAAT4-expressing regions could result in morphological changes of BG processes surrounding PF-PC synapses. EAAT4 expression increases after birth and peaks around the third week of age (Furuta et al., 1997), which parallels the maturation of BG ensheathment to PC synapses (Lippman et al., 2008). Whether EAAT4 modulates the calcium signals in BG and glial coverage around active synapses would be interesting to explore in future studies. In addition, differential activation of BG AMPARs could influence D-serine release that has been suggested to regulate PF LTD (Kakegawa et al., 2011).

Physiological variation in EAATs regulate neuroglia signaling Both physiological and pathological stimuli can alter the expression of neuronal EAATs, including synaptic plasticity and spinocerebellar ataxia (Levenson et al., 2002; Serra et al., 2006). Variation in EAAT4 expression also contributes to vulnerability of PCs to excitotoxic cell death (Yamashita et al., 2006). Yet, the consequences of transporter upregulation or downregulation has not been clear, since most studies rely on broad-spectrum pharmacological inhibitors or complete genetic deletion. Here, we demonstrate how a mere twofold difference in EAAT4 expression alters transmitter levels beyond the synapse. We speculate that the perisomatic localization and its high affinity enables EAAT4 to tightly control extrasynaptic signaling. Together, these results highlight the importance of endogenous neuronal transporter levels in neuroglial signaling and the potential functional signif- icance of transporter regulation during physiological or pathological stimuli.

\section{References}

Aoyama K, Suh SW, Hamby AM, Liu J, Chan WY, Chen Y, Swanson RA (2006) Neuronal glutathione deficiency and age-dependent neurodegeneration in the EAAC1 deficient mouse. Nat Neurosci 9:119-126.

Apps R, Hawkes R (2009) Cerebellar cortical organization: a one-map hypothesis. Nat Rev Neurosci 10:670-681.

Bergles DE, Dzubay JA, Jahr CE (1997) Glutamate transporter currents in Bergmann glial cells follow the time course of extrasynaptic glutamate. Proc Natl Acad Sci U S A 94:14821-14825.

Brasnjo G, Otis TS (2001) Neuronal glutamate transporters control activation of postsynaptic metabotropic glutamate receptors and influence cerebellar long-term depression. Neuron 31:607-616.

Brasnjo G, Otis TS (2004) Isolation of glutamate transport-coupled charge flux and estimation of glutamate uptake at the climbing fiber-Purkinje cell synapse. Proc Natl Acad Sci U S A 101:6273-6278.

Clark BA, and Barbour B (1997) Currents evoked in Bergmann glial cells by parallel fibre stimulation in rat cerebellar slices. J Physiol (Lond) 502:335-350.

Clements JD (1996) Transmitter timecourse in the synaptic cleft: its role in central synaptic function. Trends Neurosci 19:163-171.

Dehnes Y, Chaudhry FA, Ullensvang K, Lehre KP, Storm-Mathisen J, Danbolt NC (1998) The glutamate transporter EAAT4 in rat cerebellar Purkinje cells: a glutamate-gated chloride channel concentrated near the synapse in parts of the dendritic membrane facing astroglia. J Neurosci $18: 3606-3619$.

Diamond JS (2001) Neuronal glutamate transporters limit activation of NMDA receptors by neurotransmitter spillover on CA1 pyramidal cells. J Neurosci 21:8328-8338.

Diamond JS, Jahr CE (1997) Transporters buffer synaptically released glutamate on a submillisecond time scale. J Neurosci 17:4672-4687.

Dodt HU, Eder M, Schierloh A, Zieglgänsberger W (2002) Infrared-guided laser stimulation of neurons in brain slices. Sci STKE 120:PL2.

Foster KA, Crowley JJ, Regehr WG (2005) The influence of multivesicular release and postsynaptic receptor saturation on transmission at granule cell to Purkinje cell synapses. J Neurosci 25:11655-11665.

Furuta A, Rothstein JD, Martin LJ (1997) Glutamate transporter protein subtypes are expressed differentially during rat CNS development. J Neurosci 17:8363-8375.

Gong S, Zheng C, Doughty ML, Losos K, Didkovsky N, Schambra UB, Nowak NJ, Joyner A, Leblanc G, Hatten ME, Heintz N (2003) A gene expression atlas of the central nervous system based on bacterial artificial chromosomes. Nature 425:917-925.

Huang YH, Dykes-Hoberg M, Tanaka K, Rothstein JD, Bergles DE (2004a) Climbing fiber activation of EAAT4 transporters and kainate receptors in cerebellar Purkinje cells. J Neurosci 24:103-111.

Huang YH, Sinha SR, Tanaka K, Rothstein JD, Bergles DE (2004b) Astrocyte glutamate transporters regulate metabotropic glutamate receptor-mediated excitation of hippocampal interneurons. J Neurosci 24:4551-4559.

Iino M, Goto K, Kakegawa W, Okado H, Sudo M, Ishiuchi S, Miwa A, Takayasu Y, Saito I, Tsuzuki K, Ozawa S (2001) Glia-synapse interaction through Ca2+-permeable AMPA receptors in Bergmann glia. Science 292:926-929.

Ikeda Y, Dick KA, Weatherspoon MR, Gincel D, Armbrust KR, Dalton JC, Stevanin G, Dürr A, Zühlke C, Bürk K, Clark HB, Brice A, Rothstein JD, Schut LJ, Day JW, Ranum LP (2006) Spectrin mutations cause spinocerebellar ataxia type 5. Nat Genet 38:184-190.

Ishiuchi S, Tsuzuki K, Yamada N, Okado H, Miwa A, Kuromi H, Yokoo H, Nakazato Y, Sasaki T, Ozawa S (2001) Extension of glial processes by activation of $\mathrm{Ca} 2+$-permeable AMPA receptor channels. Neuroreport 12:745-748.

Kakegawa W, Miyoshi Y, Hamase K, Matsuda S, Matsuda K, Kohda K, Emi K, Motohashi J, Konno R, Zaitsu K, Yuzaki M (2011) D-serine regulates cerebellar LTD and motor coordination through the delta2 glutamate receptor. Nat Neurosci 14:603-611.

Lehre KP, Danbolt NC (1998) The number of glutamate transporter subtype molecules at glutamatergic synapses: chemical and stereological quantification in young adult rat brain. J Neurosci 18:8751-8757.

Levenson J, Weeber E, Selcher J, Kategaya L, Sweatt J, Eskin A (2002) Long- 
term potentiation and contextual fear conditioning increase neuronal glutamate uptake. Nat Neurosci 5:155-161.

Lippman JJ, Lordkipanidze T, Buell ME, Yoon SO, Dunaevsky A (2008) Morphogenesis and regulation of Bergmann glial processes during Purkinje cell dendritic spine ensheathment and synaptogenesis. Glia 56:1463-1477.

Mathews GC, Diamond JS (2003) Neuronal glutamate uptake contributes to GABA synthesis and inhibitory synaptic strength. J Neurosci 23:2040-2048.

Matsui K, Jahr CE (2003) Ectopic release of synaptic vesicles. Neuron 40:1173-1183.

Nagao S, Kwak S, Kanazawa I (1997) EAAT4, a glutamate transporter with properties of a chloride channel, is predominantly localized in Purkinje cell dendrites, and forms parasagittal compartments in rat cerebellum. Neuroscience 78:929-933.

Nikkuni O, Takayasu Y, Iino M, Tanaka K, Ozawa S (2007) Facilitated activation of metabotropic glutamate receptors in cerebellar Purkinje cells in glutamate transporter EAAT4-deficient mice. Neurosci Res 59:296-303.

Nimmerjahn A, Mukamel EA, Schnitzer MJ (2009) Motor behavior activates Bergmann glial networks. Neuron 62:400-412.

Paukert M, Huang YH, Tanaka K, Rothstein JD, Bergles DE (2010) Zones of enhanced glutamate release from climbing fiber in the mammalian cerebellum. J Neurosci 30:7290-7299.

Piet R, Jahr CE (2007) Glutamatergic and purinergic receptor-mediated calcium transients in Bergmann glial cells. J Neurosci 27:4027-4035.

Rothstein JD, Dykes-Hoberg M, Pardo CA, Bristol LA, Jin L, Kuncl RW, Kanai Y, Hediger MA, Wang Y, Schielke JP, Welty DF (1996) Knockout of glutamate transporters reveals a major role for astroglial transport in excitotoxicity and clearance of glutamate. Neuron 16:675-686.

Rudolph S, Overstreet-Wadiche L, Wadiche JI (2011) Desynchronization of multivesicular release enhances Purkinje cell output. Neuron 70:991-1004.

Scimemi A, Tian H, Diamond JS (2009) Neuronal transporters regulate glutamate clearance, NMDA receptor activation, and synaptic plasticity in the hippocampus. J Neurosci 29:14581-14595.

Sepkuty JP, Cohen AS, Eccles C, Rafiq A, Behar K, Ganel R, Coulter DA, Rothstein JD (2002) A neuronal glutamate transporter contributes to neurotransmitter GABA synthesis and epilepsy. J Neurosci 22:6372-6379.

Serra HG, Byam CE, Lande JD, Tousey SK, Zoghbi HY, Orr HT (2004) Gene profiling links SCA1 pathophysiology to glutamate signaling in Purkinje cells of transgenic mice. Hum Mol Genet 13:2535-2543.

Serra HG, Duvick L, Zu T, Carlson K, Stevens S, Jorgensen N, Lysholm A, Burright E, Zoghbi HY, Clark HB, Andresen JM, Orr HT (2006) RORalpha-mediated Purkinje cell development determines disease severity in adult SCA1 mice. Cell 127:697-708.
Takayasu Y, Iino M, Ozawa S (2004) Roles of glutamate transporters in shaping excitatory synaptic currents in cerebellar Purkinje cells. Eur J Neurosci 19:1285-1295.

Takayasu Y, Iino M, Kakegawa W, Maeno H, Watase K, Wada K, Yanagihara D, Miyazaki T, Komine O, Watanabe M, Tanaka K, Ozawa S (2005) Differential roles of glial and neuronal glutamate transporters in Purkinje cell synapses. J Neurosci 25:8788-8793.

Takayasu Y, Iino M, Takatsuru Y, Tanaka K, Ozawa S (2009) Functions of glutamate transporters in cerebellar Purkinje cell synapses. Acta Physiol (Oxf) 197:1-12.

Tanaka K, Watase K, Manabe T, Yamada K, Watanabe M, Takahashi K, Iwama H, Nishikawa T, Ichihara N, Kikuchi T, Okuyama S, Kawashima N, Hori S, Takimoto M, Wada K (1997) Epilepsy and exacerbation of brain injury in mice lacking the glutamate transporter GLT-1. Science 276:1699-1702.

Tsukada S, Iino M, Takayasu Y, Shimamoto K, Ozawa S (2005) Effects of a novel glutamate transporter blocker, $(2 S, 3 S)$-3-[3-[4-(trifluoromethyl)benzoylamino]benzyloxy]aspartate (TFB-TBOA), on activities of hippocampal neurons. Neuropharmacology 48:479-491.

Tzingounis AV, Wadiche JI (2007) Glutamate transporters: confining runaway excitation by shaping synaptic transmission. Nat Rev Neurosci 8:935-947.

Veruki ML, Mørkve SH, Hartveit E (2006) Activation of a presynaptic glutamate transporter regulates synaptic transmission through electrical signaling. Nat Neurosci 9:1388-1396.

Voogd J, Pardoe J, Ruigrok TJ, Apps R (2003) The distribution of climbing and mossy fiber collateral branches from the copula pyramidis and the paramedian lobule: congruence of climbing fiber cortical zones and the pattern of zebrin banding within the rat cerebellum. J Neurosci 23:4645-4656.

Wadiche JI, Jahr CE (2005) Patterned expression of Purkinje cell glutamate transporters controls synaptic plasticity. Nat Neurosci 8:1329-1334.

Wadiche JI, Tzingounis AV, Jahr CE (2006) Intrinsic kinetics determine the time course of neuronal synaptic transporter currents. Proc Natl Acad Sci U S A 103:1083-1087.

Wersinger E, Schwab Y, Sahel JA, Rendon A, Pow DV, Picaud S, Roux M) (2006) The glutamate transporter EAAT5 works as a presynaptic receptor in mouse rod bipolar cells. J Physiol 577:221-234.

Yamashita A, Makita K, Kuroiwa T, Tanaka K (2006) Glutamate transporters GLAST and EAAT4 regulate postischemic Purkinje cell death: an in vivo study using a cardiac arrest model in mice lacking GLAST or EAAT4. Neurosci Res 55:264-270.

Zheng K, Scimemi A, Rusakov DA (2008) Receptor actions of synaptically released glutamate: the role of transporters on the scale from nanometers to microns. Biophys J 95:4584-4596. 